\title{
Experience of Diagnostic Coding In Irish General Practice: The Practice Perspective
}

\author{
Conor Kennedy, Casey O’Brien and Claire Collins
}

Irish College of General Practitioners, Dublin, Ireland

Correspondence should be addressed to: Claire Collins; claire.collins@icgp.ie

Received date: 24 October 2013; Accepted date: 14 February 2014; Published date: 17 July 2014

Academic Editor: Elizabeth Jean Comino

Copyright (C 2014. Conor Kennedy, Casey O’Brien and Claire Collins. Distributed under Creative Commons CC-BY 3.0

\begin{abstract}
Background: Coordinated research and diagnostic coding are both in their infancy in Irish general practice. As part of a larger project, which aimed to investigate the feasibility of primary diagnostic ICPC-2 coding in the Republic of Ireland with a view to creating a national morbidity database via the introduction of coding to a sample of computerised practices, a survey of GP experiences in this regard was undertaken. Research Question: What are the challenges, barriers and facilitators experienced by participating GP practices in terms of establishing diagnostic coding in their practice? Methods: An online survey of participating practices $(n=25)$, including detailed open-ended responses, was carried out. Results: According to respondents, the most significant barriers to the implementation of diagnostic coding in Irish general practice include the impact on consultations with regard to time, patient care, the limitations (and resultant frustrations) of the practice management software (PMS) systems and the ICPC-2 classification structures. Respondents displayed a high level of competence in terms of using their PMS, a willingness to contribute towards the overall goal of a national morbidity register, a degree of flexibility in terms of adopting the initial additional workload, and perseverance in the knowledge of the existence of a learning curve in terms of diagnostic coding. Conclusions: There exist both technical and cultural barriers to the establishment of coding throughout Ireland; including issues in relation to the time required to code, and concerns about the specificity of the codes being used to adequately record morbidity. However, it is evident that the skills and desire exist to overcome these obstacles with a view to improving practice and patient management.
\end{abstract}

Keywords: General practice, diagnostic coding, data management, ICPC-2.

\section{Introduction}

General practitioners (GPs) and health authorities have integral roles in assessing the needs of their local populations and providing services to meet these needs (Pearson et al, 1996). This requires current information on the incidence and prevalence of disease in general practice, and not surprisingly the demand for this information has increased. In settings such as the UK (CPRD, 2013), Netherlands (NIVEL, 2013), and Australia (Britt et al, 1999) databases of general practice information provide a rich source of population health data. In Ireland, while 
the use of information technology has increased with computerisation of general practice, (reported as $89 \%$ in 2005 by O'Dowd et al, 2006), the use of diagnostic coding is not widespread. There is an immediate need for information on the incidence, and prevalence of acute and chronic conditions in Irish general practice. Available survey data indicate that $51 \%$ of Irish adults have visited a medical doctor or hospital in the previous 12-months, and that nearly one-third of these are on longterm medication for conditions such as blood pressure, arthritis, asthma, diabetes, heart disease and cancer (Irish Patient Association, 2004). Most of the care of chronic disease in Ireland takes place in primary care (Department of Health and Children, 2009) and it is predicted that there will be 13.4 million general practice consultations in Ireland by 2015 and 14.8 million by 2021 (Layte et al, 2009).

General practice/family medicine in Ireland is delivered largely by circa 2,500 general practitioners (GPs) operating in approximately 1,500 practices, each operating as an independent unit with 35\% of GPs working in single-handed practices. The state finances $30 \%$ of the population's primary care, and only $4 \%$ of GPs are not involved in this scheme (O'Dowd et al, 2006). The general practitioner is often the first point of contact an individual has with the health care system in Ireland, but little detail is known about who attends, the reason for attendance and the treatment path followed. As such, general practice is a largely untapped source of information about the health of the Irish population.

Access to this type of information in Ireland would assist in service delivery, service and policy planning, quality assurance and patient treatment decisions. In turn, there are benefits at consumer level as patients are likely to receive better treatment from GPs if their health needs are more precisely targeted. A further benefit is that with continuous consultation recording, the history of illness in primary care can be tracked over time resulting in greater understanding of the development of disease and continuing care. Health researchers can also benefit from the use of a central database of standardised and deidentified general practice information

Health surveys and databases often use standard classifications and terminologies to gather data from different settings. This enables information to be systematically recorded and aggregated for interpretation, analysis, and comparison across regions and time periods (de Lusignan and van Weel, 2006). These systems can also highlight patients who are eligible for specialist clinics (Midgley and O'Connell, 1998). Further, the use of standardised coding system means that with the use of mapping technology there are future opportunities for linking the data with other national databases, as well as comparing Irish general practice information with that from other countries.

Aside from relatively small scale efforts to introduce diagnostic coding in the south east of the country during the 1990s (O'Mahony et al, 2001), the entire concept of classification in general practice, while familiar to most, is most certainly in its infancy in Ireland. Scepticism about the value of coding consultations is widespread; and the previous concerns regarding the intrusion into the consultation and the inadequacies of computerisation in Irish general practice are still relevant (Hunter, 2005).

As part of a larger project which aimed to assess the feasibility of both incorporating diagnostic coding into routine Irish general practice, and the development of a general practice morbidity and epidemiological database, a survey of the 25 participating practices, who received training and used the ICPC-2 classification system to code their consultations over an 18 month period, was carried out. This survey aimed to gain an understanding of practice experiences of implementing coding procedures including a consideration of the challenges faced, satisfaction with training received, and their willingness to code in the future. 


\section{Methodology}

Employing both quantitative and qualitative methods, an online survey was conducted using surveymonkey.com. The aim of the survey was to establish the experience of the 25 study practices in a range of aspects in the project. All twentyfive practices taking part in the GPMED study were contacted by email and asked to complete the survey online. The email was sent to the project lead in each practice, who in most cases was the principle general practitioner (GP). Respondents were asked to incorporate feedback from all practice staff when replying to the survey. Respondents were given the opportunity to give open-ended responses to some questions and to elaborate on the answers selected.

The findings below reflect the views of seventeen study practices who took part. The data received were exported from surveymonkey.com to SPSS where a descriptive analysis was conducted. Qualitative data were imported to Nvivo where a thematic analysis was conducted (Braun \& Clarke, 2006).

Ethical approval to undertake this study was sought and received from the Ethics Committee of the Irish College of General Practitioners.

\section{Results}

Principal GPs $(\mathrm{n}=15)$, other practice GPs $(n=10)$, and Practice Nurses $(n=12)$ were involved in coding in the participating practices.

Most $(n=13)$ of the respondents explicitly recognised that coding was a vital tool in order to achieve a better knowledge of the day-to-day problems being dealt with in a busy practice:

"(It enables me) to gather lists of patients for statistics, communication, audit, etc..."

The practicing of routine coding was also seen to enable the creation of databases on some of the most prevalent diseases presenting to practices, offering the opportunity for better patient management:

"We have instant registers for diabetes, hypertension, asthma, renal disease etc. We are also able to know, at any one time, who is on a particular medication."

“...it enables us to form morbidity registers - especially useful for getting lists of patients together for immunisation against flu etc..."

A key element noted by respondents is the "quick reference" function offered by such reports enabling the practitioner to assess the patient need in an instant.

Respondents were asked to identify the main challenges or obstacles which hampered their progress in developing coding in their practices. Finding time to code is the most reported challenge facing practices, and choosing the correct code to enter for the consultation is the second most reported challenge.

The majority of respondents $(n=12)$ stated that the coding of each consultation took less than sixty seconds to complete, a further four respondents stated that coding took 1-2 minutes, while one respondent said they spent 2-5 minutes coding a consultation.

Almost two-thirds of respondents stated that the coding of the consultation is conducted during the consultation itself. The remaining third opted to code the consultation immediately after the patient had left the surgery.

Respondents displayed a level of frustration in the organisation of the coding structures stating that they found the ICPC2 codes to be "too vague" and "broad" in terms of diagnosis, while some felt that these attributes of rigidity meant that the codes became "very cumbersome" and that "the codes do not always match up" to what is being seen in consultation:

"The codes are not intuitive - indeed they are probably out of date as conditions which were common when the codes were 
devised are no longer common, and now more common conditions do not have their own code."

When asked to indicate the suitability of ICPC-2 for coding the reason for encounter, symptom and diagnosis of each consultation, twelve respondents found ICPC-2 coding usually or always suitable for the diagnosis; however, this was only the case for symptom and reason for encounter in less than one out of three instances (Table 1).

Table 1: Suitability of ICPC-2 coding

\begin{tabular}{|l|l|l|l|}
\hline & Not/Rarely & Sometimes & Usually/Always \\
\hline Reason for encounter & 4 & 8 & 5 \\
\hline Symptom & 6 & 6 & 5 \\
\hline Diagnosis & - & 5 & 12 \\
\hline
\end{tabular}

Many noted the ease of use that came over time, and that coding during a consultation can become very "quick if a familiar condition (presents)"as the practitioner comes to remember the most frequently used codes; "I now know most of the codes of the chronic diseases". Respondents also noted that an understanding of how ICPC is constructed is of importance:

"...knowing that 1-30 are symptom items and 70-100 are diagnosis items....and that they are broken into systems helps".

Some respondents also favoured the approach taken in this study in terms of limiting the number of diagnoses to be coded instead of coding all diagnoses seen, and described how this approach has meant that the introduction to coding has been more manageable; "only a few diagnoses are coded currently so (I) only have to do it for certain ones so (I) can remember the codes (which) makes it easier to add to the records". As a result, thirteen respondents rated themselves as "moderately" or "very" competent in the use of ICPC-2.

No practice reported that coding diagnoses using ICPC-2 had a negative impact on their routine use of their practice management software; eleven respondents stated that it had no impact, and six said it had a positive impact. The positive impacts noted were in terms of getting greater use from, and understanding of their practice management software system. Practices noted that involvement in this study has provided a "more rigorous approach to coding" and has resulted in "staff thinking more about stating the diagnosis" from a consultation.

Table 2 shows the responses with regard to the ease with which the practice management software system permitted the recording of various aspects of the consultation. The proficiency of those questioned in relation to their practice management system (PMS) is evident from the finding that most respondents found it very easy/easy to record most aspects of the consultation. The only aspect of the consultation, that seemed to prove challenging in terms of recording, was in relation to the ordering of tests using their systems with half of those who responded to this question describing this process as difficult/very difficult. 
Table 2: Ease of recording aspect of the consultation in the practice management software system

\begin{tabular}{|l|l|l|l|}
\hline & Very Easy/Easy & $\begin{array}{l}\text { Difficult/Very } \\
\text { Difficult }\end{array}$ & Not applicable \\
\hline $\begin{array}{l}\text { Reason for } \\
\text { Encounter }\end{array}$ & 7 & 5 & 2 \\
\hline Symptom & 7 & 6 & 1 \\
\hline Diagnosis & 9 & 5 & 0 \\
\hline Treatment & 10 & 3 & 1 \\
\hline Medication & 10 & 2 & 2 \\
\hline Referral & 10 & 3 & 1 \\
\hline Procedures & 8 & 5 & 1 \\
\hline Tests ordered & 6 & 6 & 1 \\
\hline Test results & 8 & 4 & 1 \\
\hline
\end{tabular}

Greater integration of coding for research into the PMS appears to be the key to improving the feasibility of same in day-today practice. Thirteen of the seventeen respondents expressed that the feasibility of this would improve if their PMS had specific fields to accommodate the specified study variables, while ten respondents felt that feasibility would be improved if the software offered better support for ICPC-2 coding.

When asked for further opinion, some respondents echoed earlier expressed concerns about the relevancy of the codes in terms of "conditions which are seen in modern day Irish general practice", while others spoke of their view that the key to increasing participation in coding is the production of tangible and relevant results in, for example, report format specific to the practice.

Overall, ten respondents had made an attempt to extract their ICPC-2 coded data from their PMS. The extraction of data was totally successful in only two cases, while six found it to be only moderately successful or unsuccessful. Six respondents provided further detail on this reporting difficulties based around extracting "in a consistent manner", ensuring that the same patients do not appear repeatedly on the same report and "bad software design" which hampers extraction efforts. Three quarters of those who were able to extract data from their practice management systems detailed that this data were useful to them and their practice.

Thirteen respondents reported that apart from the data specific to the GPMED study, they retrieve data at regular intervals with one doing so at least annually, seven doing so on a monthly basis and five doing so at least weekly.

Reflecting the wide range of possibilities available with consistently maintained registers, respondents offered a wide range of differing ways in which they use the data they extract. In the main, the need to monitor "patients with a particular diagnosis or on a particular medication" accounted for most of the information being sought. Also mentioned is the current need to identify those in the risk groups who have been targeted to receive the influenza vaccination, while others stated that information had been extracted from their systems in order to compile "inhouse reports" and for completing "audits of various aspects of practice for example referrals".

Over half of those who responded to this question stated that they did not encounter any substantial difficulties in producing the information they sought. However, it is apparent that in cases it can be "difficult to retrieve" the correct data. Aside from this process being "tedious" at times, some also stated that "for basic things it is easy" however, when greater detail is required, problems may arise. In this instance a 
certain level of knowledge of the system and skill in report production is required.

\section{Discussion}

Despite, almost three quarters of respondents detailing that the coding of each consultation required less than sixty seconds to complete (and the remainder not taking more than two minutes to code), finding time to code either during a patient consultation, or overall during a busy working day, was the most reported challenge facing practices in their endeavour to develop their coding capacity. Concern exists, as is noted in similar studies in the UK, about the "inevitable trade-off between the time devoted to the computer... at the expense of giving time and attention to the patient" (de Lusignan, 2005:92).

Further, probing suggests that the patient management software being used may be partly to blame also with one third of respondents describing the recording of diagnosis and symptom as "difficult". While, most respondents detailed a high level of proficiency in the use of their software, with half calling its everyday use "easy" or "very easy", the customer/provider relationship can be understood to be somewhat strained with notable disparities between consumer expectations and actual service provision. This is especially the case for some of those using the relatively older systems who lament the fact that there exists "no prospect of the software vendors taking out the information (being coded/entered) for research purposes", creating doubt over the value being added to, or indeed lost from the practice by coding. Further, over half of those questioned, working with both old and new systems, felt it necessary for the software providers to offer better support for, and understanding of ICPC-2 coding requirements in their practice. This level of ambiguity and confusion and lack of relevant technical support inevitably contributes further to the issue of time highlighted by most respondents.

The integrity of the data may also be seen to be put at risk due to the above issues.
Respondents who encountered the above difficulties during their attempt to code a consultation, out of consideration for their patients, were often reluctant to use consultation time to overcome a particular obstacle in the process. Thus, some practitioners acting in good faith in attempting to code each consultation would resort to entering "the nearest match" to the originally desired code for the condition(s) being treated. Feedback on how to counter this echoed the findings of Gray et al (1989) in their examination of coding using ICHPPC-2-Defined codes, with some practices mentioning the need for training workshops for coders focussing on the main sources of error.

Taken, then, as a package of concerns, including the results stated above detailing the perceived inadequacies of ICPC-2 classification, there exists a myriad of factors that may serve to undermine efforts made by project leads in each practice in "trying to teach, encourage and maintain usage of coding by all (practice) staff, on a consistent basis".

Efforts have been made to address these issues; however, further progress is required. ICPC-3 is in development at present with an unknown publication date at the time of writing. Following dialogue with practitioners and interest groups, updated versions of patient/practice management systems with an enhanced data recording and searching capabilities, have begun to enter the market in Ireland, a trend that will continue at pace with the development of new systems in this area. Also, papers focussed on providing details of the possibilities, and examples of the tangible results have been published and disseminated widely in Ireland in order to stimulate a discourse, promote the relevance of coding and advise on the potential that exists (Collins and Kennedy, 2009).

Acknowledgement of a learning curve with respect to coding of consultations, as similarly noted by Meade (2005) in relation to the computerisation of practices in general, is also evident from findings. It seems that the advance of time itself may 
contribute to resolving some of the above difficulties. When asked to detail the easiest aspects of coding their consultations, it is apparent from the responses, as noted above, that gaining familiarity with the coding mechanism of the particular software system and with the codes themselves is crucial to the facilitation of coding in general practices.

Contrary to the sentiments recorded in Britain (de Lusignan, 2005), the mood of the respondents was mostly constructive and motivated with responses littered with comments, suggestions and criticisms aimed at making the entire process more workable and efficient. Most respondents expressed a preoccupation with completing the process in the correct manner. When asked about the difficulties encountered when extracting data, similar to responses given relating to data entry, most respondents reported difficulties in extracting data "in a consistent manner".

In spite of the above issues, however, the impact of coding for this study has not been negative on any participating practice. No practices reported that taking part in the project had a negative impact on their use of their practice management software. Two thirds of respondents stated that using ICPC-2 coding had no impact on their routine use of their practice software while, the remaining third of respondents felt that the impact of the study had been positive in

\section{References}

1. Braun, V. and Clarke, V. (2006) "Using thematic analysis in psychology," Qualitative Research in Psychology, 3 (2) 77-101.

2. Britt, H., Sayer, G.P., Miller, G.C., Charles, J., Scahill, S., Horn, F., and Bhasale, A. BEACH Bettering the Evaluation And Care of Health A study of general practice activity, six-month interim report. AIHW cat. no. GEP 1. Canberra: Australian Institute of Health and Welfare (General Practice Series no. 1). terms of getting greater use from, and understanding of their systems. When asked in broader terms about the overall burden of the project, the majority felt that coding had positively impacted in a number of ways; by providing a "more rigorous approach to", and uncovering "the power of coding". Others described this project in terms of a thought provoking introduction to research in primary care in Ireland, and acknowledged the potential to develop this aspect within the constraints of day-to-day general practice; "a lot can be achieved through studies like this (by) opening one's mind".

Crucially, this study has found that, among this sample, there is an overwhelming acceptance of the legitimacy of coding as the accepted way forward in the data management aspect of general practice, and an understanding of the potential value that lies within this. Future work should aim to address the concerns of practitioners raised here by considering methods to reduce the time requirements of coding, improve practice management software towards facilitating this coding outcome, and use coded data to produce work discussing practice specific and community salient issues to heighten awareness of the value of primary care data collection, and extraction to individual GPS and practice management software providers.

3. Collins, C. and Kennedy, C. (2009) "Reaping the benefits of diagnostic coding," Forum, 26 (12) 18.

4. CPRD. The Clinical Practice Research Datalink. [Online], Medicines and Healthcare Products Regulatory Agency. [Accessed October 21, 2013], Available: http://www.cprd.com/intro.asp

5. de Lusignan, S. (2005) "The barriers to clinical coding in general practice: a literature review," Medical informatics and the internet in medicine, 30 (2) 89-97.

6. de Lusignan, S. and van Weel, C. (2006) "The use of routinely collected computer data for research in primary care: opportunities and challenges," Family 
Practice, 2006 Apr; 23 (2) 253-63. Epub 2005 Dec

20.

7. Department of Health and Children. (2009) Health in Ireland: Key Trends 2009. Dublin: Department of Health and Children.

8. Gray, D., Ward, A., Underwood, P., Fatovich, B., and Winkler, R. (1989) "Morbidity coding in general practice," Family Practice, 6 (2) 92-97.

9. Hunter, N. (2005) "Computers - boon or bugbear for GPs?” Forum, 22 (2) 12-14.

10. Irish Patient Association and Lansdowne Market Research. (2004) Patient Attitudes. Dublin: Irish Patient Association.

11. Layte, R., Barry, M., Bennett, K., Brick, A., Morgenroth, E., Normand, C., O' Reilly, J., Thomas, J., Tilson, L., Wiley, M., Wren, M.A. (2009) Projecting the impact of demographic change on the demand for and the delivery of health care in Ireland. Research Series No. 13. Dublin: Economic and Social Research Institute.

12. Meade, B. (2005) "In defence of practice computerisation," Forum, 22 (3) 16-17.
13. Midgley, A. and O'Connell, S. (1998). Read Codes. In. O'Connell (Ed.). Handbook for Non-Principals in General Practice. [Online] National Association of NonPrincipals West Midlands NHS Executive. [Accessed February 11, 2014]. Available: http://www.nasgp.org.uk/handbook/33.ht $\mathrm{m}$

14. NIVEL - Netherlands Institute for Health Services Research. [Online] [Accessed October 21, 2013] Available: http://www.nivel.nl

15. O’Dowd, T., O'Kelly, M., and O'Kelly, F. (2006) Structure of General Practice in Ireland 1982-2005. Dublin: Irish College of General Practitioners and Trinity College Dublin.

16. O'Mahony, B., O'Neill, Y., Lynch, C., Fennessy, M., Lanigan, A.M., and O'Reilly, 0. (2001) "Morbidity Data Collection in General Practice: Experience in the South Eastern Health Board 1998-1999," Irish Medical Journal, 94 (10) 299-302.

17. Pearson, N., O’Brien, J., Thomas, H., Ewings, P., Gallier, L, and Bussey, A. (1996) "Collecting morbidity data in general practice: the Somerset morbidity project," British Medical Journal, 312 (7045) 1517 1520. 